\title{
An Automated Tool for Non-contact, Real Time Early Detection of Diabetes by Computer Vision
}

\author{
Jamal Firmat Banzi, Member, SCIEI and Zhaojun Xue
}

\begin{abstract}
There has been considerable progress in computer vision, artificial neural network and pattern recognition in the last two decades, and there has also much progress in medical imaging technology in recent years. Although images in digital form can be processed by basic image processing techniques, effective use of computer vision can provide much useful information for diagnosis and treatment. In this paper we integrate computer vision and iridology practice for the detection of diabetes. Using iridology iris image is evaluated by detecting the presence of broken tissues and change in color pattern. According to iridology the abnormality in an iris of the human eye represent the abnormality of the corresponding organ conferred by the iris chart. In this research we examine pancreas organ which is at position 01:45 - 02:15 for the right eye and 07:15-7:45 for the left eye according to Dr. Jensen iris chart. We applied two methods to reach our conclusion, visual inspection method and color coding method. The artificial neural network is used for training and classification purpose. The entire process is showing a high accuracy detection of abnormality of pancreas organ which led to diabetes. The final result is compared with the insulin normality test for verification.
\end{abstract}

Index Terms - Computer vision, diabetic, feature extraction, iris, iridodiagnosis.

\section{INTRODUCTION}

Development of computer technology and services led to an increased efficiency, highly reliable, fast and accurate diagnosis and treatment of the most dangerous diseases. Most of convectional diagnosis methods involve puncturing of body tissue (skin) to get blood sample for examination. However there are alternative diagnosis methods presented in iridology. Iridology, also known as iridodiagnosis, is an alternative medicine practice in which patterns, colors, and other characteristics of the iris are examined for information about a patient's systemic health [1].

The science of iridology is the science of analyzing the fibers colors and signs in the eye to glean this information about imbalances in the body, where they are and how severe they are [1].

The iris is actually part of the nervous system and its intricate highway of hundreds of thousands of nerve endings and nerve impulses. The iris is a forward continuation of the choroid coat [1]. The iris receives its name (iris, irides,-a rainbow) from its varying color effects. The nerve fibers in the iris of the eye receive their impulses from the rest of the body through the optic nerve, optic thalami and spinal cord [1].

Manuscript received June 12, 2014; revised January 9, 2015.

The authors are with Tianjin University, Tianjin, China (e-mail: jamalbanzi@yahoo.com).
Iridology can be integrated with finest technology such as computer vision for an accurate identification of abnormality in different organs of a human body. The computer vision is the contemporary technology which involves transformation of data from a still or video camera into either a decision or a new representation for the purpose of achieving some particular goal [2]. One of the most prominent application field of computer vision is medical imaging. This area is characterized by the extraction of information from image data for the purpose of making a medical diagnosis of a patient.

In this research, Computer vision is linked with iridology to examine patient's iris for tissue change, as well as features such as specific pigment patterns and predict diabetes status of a patient. With computer vision, a change in color pattern or presence of broken tissue in the iris of the eye will be examined and analyzed by image processing techniques to diagnose diabetes.

\section{RELATED WORK}

Previously detecting diseases (diagnosis) using iridology principles were done manually, this led to get an entirely negative results and raised the claim that iridology is not a useful diagnostic tool. For example in 1979 Dr. Bernard Jensen, a leading American iridologist and two other iridology proponents failed to establish the basis of their practice when they examined photographs of the eyes of 143 patients in an attempt to determine which ones had kidney impairments. Of the patients, forty-eight had been diagnosed with kidney disease, and the rest had normal kidney function. Based on their analysis of the patient's irises, the three iridologists could not detect which patients had kidney disease and which did not [1], [3].

This is one of the prevalent challenges facing iridology, the reason for such mistake can only be explain by imperfect of the human eye in observation as some iris features such as broken tissues and color pattern cannot be easily observed by a naked human eye. However these challenges can be minimized by applying computer vision technology implanted with specific algorithm for examining abnormality of the tissues in the iris.

Computer can extract deep inside features of each pixel of the iris image using image processing techniques such as image quality assessment, segmentation of iris, iris normalization, feature extraction and classification for clinical diagnosis and suggest the result with a lot higher accuracy.

The study of iridology to detect abnormality of human organs by examining iris was much improved after the inversion of computer technology which increased the 
accuracy of diagnosis. For example $\mathrm{Ma}$ and $\mathrm{Li}$ (2008) suggested texture feature extraction and classification for iris diagnosis. They proposed an iridology model that consists of the iris image pre-processing, texture feature analysis and disease classification. Their experimental result shows that the proposed iridology diagnosis model is quite effective and promising for medical diagnosis and health surveillance for both hospital and public use [3].

However and unlike the conventional method for diagnosing diabetes which are not real time and which seems to be inconvenient and discomfort to many people as it involve fasting, puncturing of the body tissue (skin) to get blood sample and which may in a way cause problems like swelling or hardness under the skin, severe pain and sometimes fever and other possible complications [4]. The proposed tool for diabetes diagnosis is non-contact, real time, portable and more convenient as it does not involve skin puncturing and it can be used both in the hospital and at home for self-diagnosis.

\section{PROPOSED APPROACH}

The main purpose of this research is to apply computer vision technology in medical imaging and subsequently design a diabetes diagnostic tool which is real time and non-contact and therefore more convenient in diagnosing diabetes. The designed tool transform an image of the iris of the eye into new representation using image processing algorithms and analyses some changes in pattern such as color pattern and broken tissues in the region of iris corresponding to pancreas organ according to iris chart and make a diagnosis whether a patient has diabetes or not. The block diagram for iris image processing algorithm is shown in the Fig. 1 below. We divided the algorithm into three stages which are;

\section{A. Pre-processing Stage}

It involves steps such as image acquisition and definition of a region of interest (ROI). Image acquisition involves all steps of acquiring image for iris image analysis. It's also known as feature extraction. In this research the main tool for taking the image of the iris is a high resolution video camera known as Logitech c920 programmed with OPENCV/C++ library.

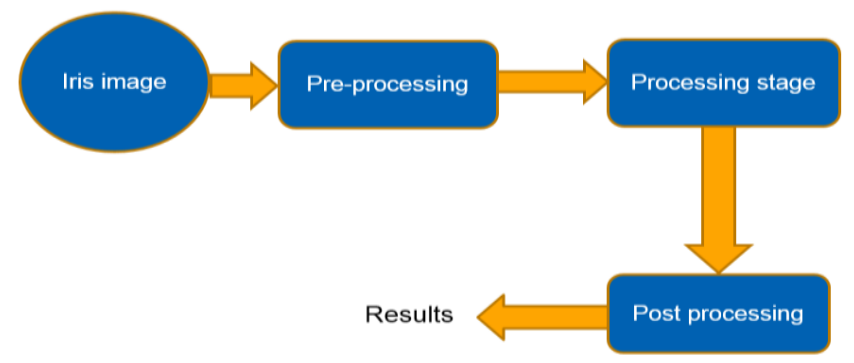

Fig. 1. Block diagram for iris image processing algorithm.

For a quality image and in order to avoid rotation of the position of the eye which can cause shifting of the area of pancreas, standard tool like holder of the head, a tripod for stabilizing the angle of the camera and a handler for hands were used. A slit lamp is also important to increase light intensity during experiment [5]. Since a processing need to be applied only on the iris of the eye and not to whole eye, defining a region of interest is important.

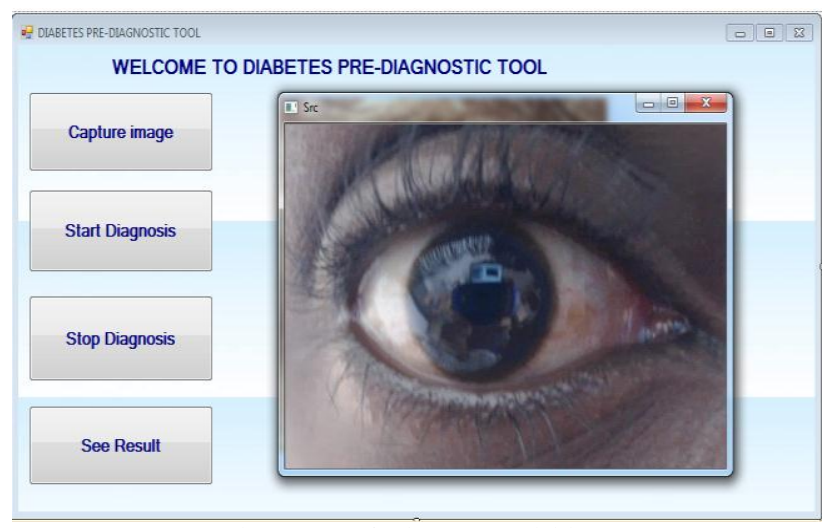

Fig. 2. GUI for image acquisition.

\section{B. Processing Stage}

The stage involve processing techniques such as Localization, Segmentation, Filtering, Normalization and enhancing the final result.

\section{1) Filtering}

Is done to remove noisy without blurring the image.

Techniques like median filter, unsharp mask, mean filter and Gabor filter were used. For better result of texture segmentation we proposed to use Circular Gabor filter defined by the following equation;

$$
G(x, y)=g(x, y) \exp \left\{2 \pi i F\left(\sqrt{\left(x^{2}+y^{2}\right)}\right)\right\}
$$

where $F$ represents the central frequency for a circular Gabor filter. The properties of the circular Gabor filters can be more explicit in their frequency domain.

The Fourier representation of the circular Gabor filter is as follows:

$$
\text { Fourier }(u, v)=\frac{\sqrt{(2 \pi)}}{2} \alpha \exp \left\{-\frac{\left(\sqrt{\left(u^{2}+v_{2}\right)}-F\right)^{2}}{2 a^{2}}\right\}
$$

where $\alpha=1 / p i S$

In case of Gabor based texture, the analysis of the texture property of every single pixel is provided by the projection of the textured surface $I(x ; y)$ onto a complex Gabor wavelet [6].

\section{2) Localization/segmentation}

John Daugman's approach is used for localization/segmentation of iris from the rest of the eye portion [7]. This step provides better accuracy for segmentation. The task of image segmentation is to partition an image into non-overlapping regions based on intensity or textural information thereby identify specific features in this case broken tissues in the region of pancreas in the iris of the eye. Since iris boundaries is not exactly circular according to some research [8], in this research an active contours approach is used, with this method a deformable model is used which evolve in each iteration to minimize a given energy function. Active contours can be performed using two methods;

Edge-based-which uses an edge detector which depends on 
image gradient to stop the evolved curve on the boundary of the desired object [8].

Region based-in which the foreground and background regions are detected. It can detect object whose boundaries are not necessarily be defined by the gradient [8]. For accurate extraction of intensity information in the local region for example to find the pupil and iris boundaries accurately the region based method is ideal.

We will see that the analysis of local regions leads to the construction of a family of local energies at each point along the curve. In order to optimize these local energies, each point is considered separately, and moves to minimize (or maximize) the energy computed in its own local region. To compute these local energies, local neighborhoods are split into local interior and local exterior by the evolving curve. The energy optimization is then done by fitting a model to each local region.

We let $I$ denote a given image defined on the domain $\Omega$, and let $C$ be a closed contour represented as the zero level set of a signed distance function $\varphi$, i.e., $C=\{x \mid \varphi(x)=0\}$.We specify the interior of $C$ by the following approximation of the smoothed Heaviside function:

$$
\delta \varphi(x)=\left\{\begin{array}{cc}
1, & \varphi(x)=0 \\
0, & |\varphi(x)|<\varepsilon \\
\frac{1}{2 \varepsilon}\left\{1+\cos \left(\frac{\pi \varphi(\chi)}{\varepsilon}\right)\right\}, & \text { otherwise }
\end{array}\right.
$$

We now define an energy functional in terms of a generic force function, $F$. Our energy is given as follows,

$$
E(\varphi)=\int_{\Omega_{X}} \delta \varphi(x) \int_{\Omega_{y}} \mathrm{~B}(x, y) \bullet F(I(y), \varphi(Y)) d y d x .
$$

The function, $F$ is a generic internal energy measure used to represent local adherence to a given model at each point along the contour.

\section{3) Normalization}

Once the iris region is segmented from an eye image, the next step is to transform the iris region into fixed dimensions. The normalization process will produce iris regions which have the same constant dimensions, so that two photographs of the same iris under different conditions will have characteristic features at the same spatial location.

For normalization of iris regions a technique based on Daugman's rubber sheet model is employed [7], [8]. The center of the pupil was considered as the reference point, and radial vectors pass through the iris region.

\section{4) Enhancement}

The basic goal of image enhancement is to process the image so that we can view and assess the visual information it contains with greater clarity and in accordance with iridological analysis.

Image enhancement techniques can be divided into two broad categories:

Spatial domain methods, which operate directly on pixels, and Frequency domain methods, which operate on the
Fourier transform of an image [8]. In this research we proposed spatial domain methods. One of the method is the histogram equalization. This is because the output of these methods can be interpreted using iridology perspective. Histogram equalization involves finding a grey scale transformation function that creates an output image with a uniform histogram (or nearly so). We must find a transformation $T$ that maps grey values $r$ in the input image $F$ to grey values $s=T(r)$ in the transformed image $\hat{F}$. It is assumed that. $\mathrm{T}$ is single valued and monotonically increasing, and

$$
0 \leq T(r) \leq 1 \quad \text { for } \quad 0 \leq r \leq 1
$$

The inverse transformation from $s$ to $r$ is given by $r=T^{-1}(s)$ and therefore after substitution the uniform distribution function is given as:

$$
P_{s}(s)=P_{r}(r) \frac{1}{P_{r}(r)}=1
$$

for all $s$, where $0 \leq s \leq 1$

Thus, $P_{s}(s)$ is now a uniform distribution function.

\section{Post processing Stage}

In this stage process like features extraction and classification are involved. The purpose of this phase is to verify the result obtained from processing stage.

If the observation shows the signs of the presence of diabetes then the image is subjected to this stage for verification.

\section{1) Feature extraction}

The region of interest is identified by visual inspection as per chart of Iridology. The dimension of the processing image reduced to $20 \times 20$ which is region of interest. Then the image was converted to matrix in order to be able to transfer it from OPENCV platform to Matlab using MEX function. Then the features for region of interest are extracted using Principal Component Analysis (PCA) [8]. The methodology was done previously for 800 subjects of which 400 are normal iris and 400 are diabetic iris. The standard images of the iris were obtained from Biometrics Ideal Test. PCA algorithm is applied on both diabetic and normal irises and features are extracted.

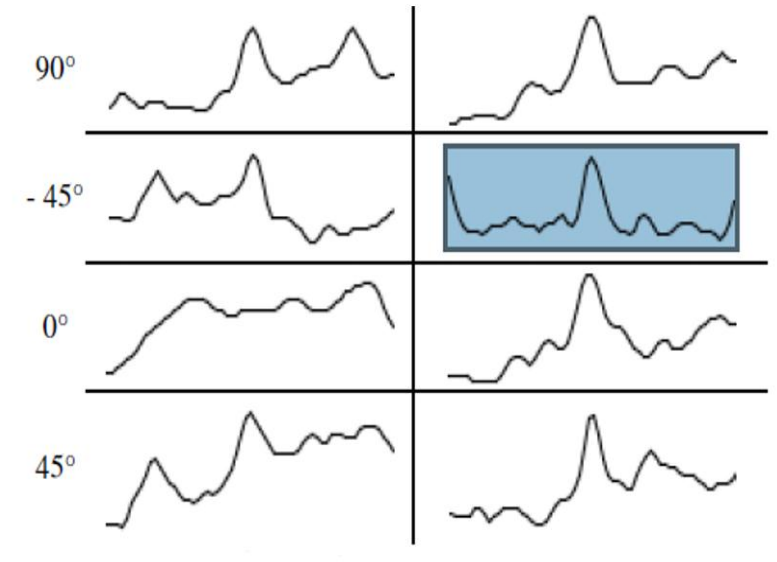

$$
\text { Normal tissues pattern }
$$

Broken tissues pattern

Fig. 3. Feature extraction showing normal tissues pattern and broken tissues pattern. 


\section{2) Classification}

For classification purpose Artificial Neural Network is used because they have a unique set of characteristics [9].

They are not programmed, instead they are trained by being repeatedly shown large numbers of examples for the problem under consideration [9].

As a result of this, they can provide good results in relatively short timescale. Fig. 3 shows feature classification using Artificial Neural Network.

The Network used for classification is feed forward back propagation and the training function that updates the weight and bias of the network is Levenberg-Marquardt back propagation [8], [9]. The initial values and different parameters like Maximum number of epochs, Performance goal and learning rate are defined by training algorithm used. Inputs for the network are features extracted from PCA and they are trained to classify among normal or diabetic subject.

\section{EXPERIMENTAL RESULTS}

In examining iris image for clinical features analysis based on iridology visual inspection, enhancement is an essential technique for extraction of profound features. For feature extraction different image enhancement methods which are spatial domain methods such as gray scale manipulation, discrete formulation, Image smoothing and histogram equalization. However in this research we directed more efforts on adaptive histogram equalization because of visual inspection based on iridology. The results for the processing stage are as shown in Fig. 4 below:

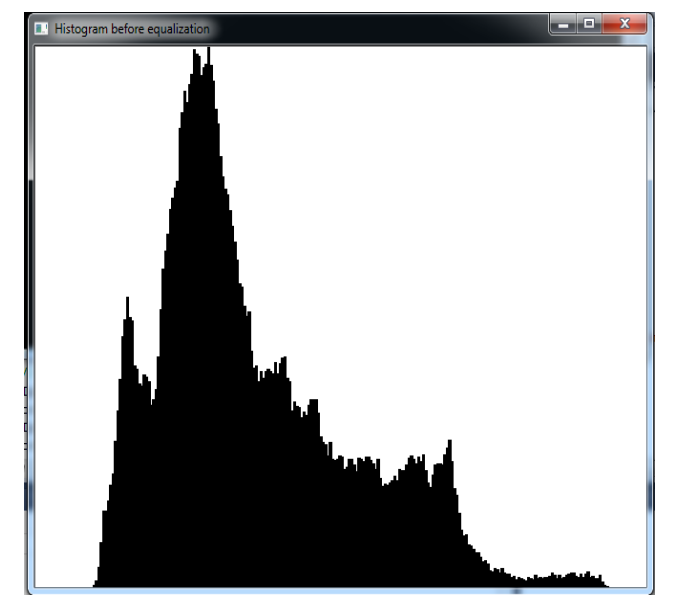

Fig. 4. (a) The Histogram of a normal iris before equalization.

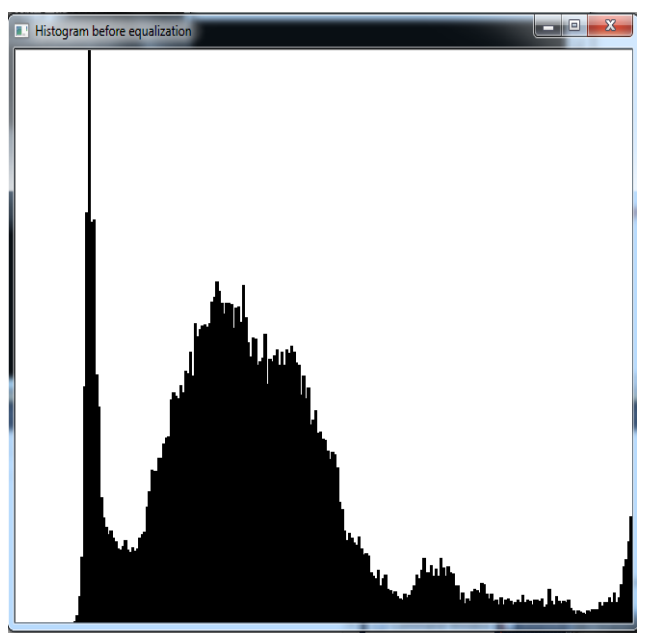

Fig. 4. (b) Histogram of a diabetic iris before equalization

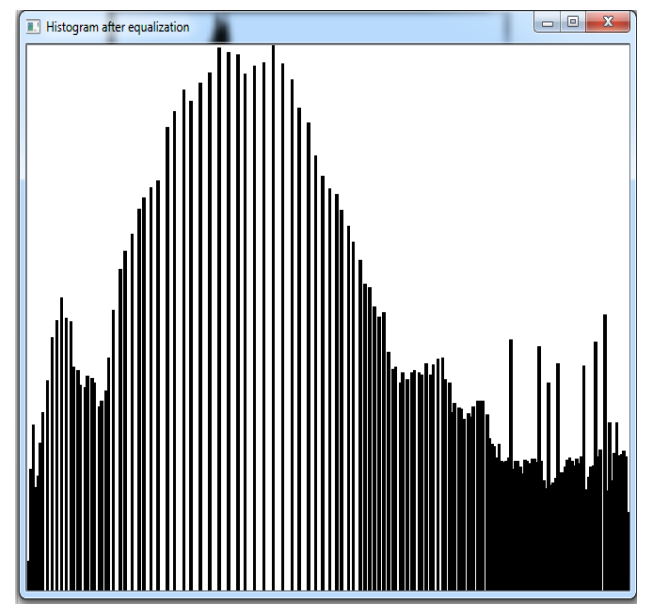

Fig. 4. (c) Histogram of a normal iris after equalization.

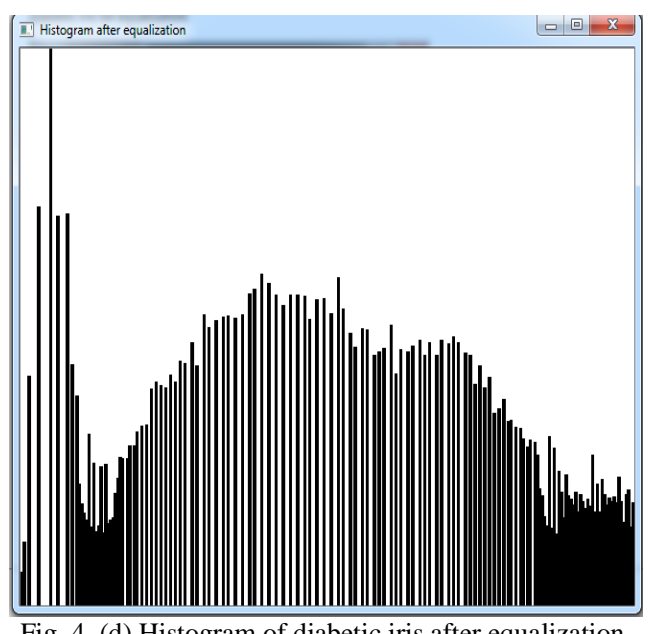

Fig. 4. (d) Histogram of diabetic iris after equalization.

For visual inspection of the region of iris corresponding to the pancreas organ which is position 01:45 to 02:15 for the right eye as for this research. The image sections after normalization are as shown in Fig. 5 below.
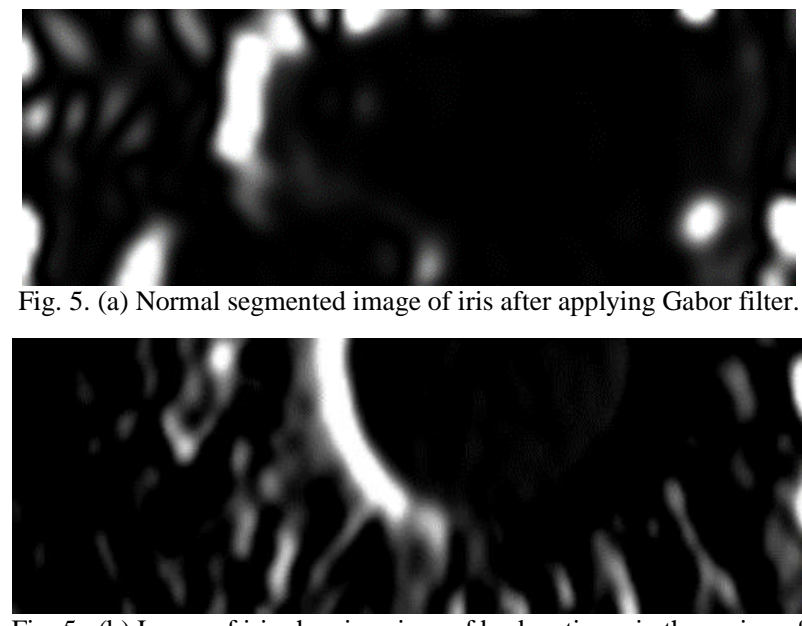

Fig. 5. (b) Image of iris showing signs of broken tissue in the region of pancreas in the iris after segmentation and application of Gabor filter.

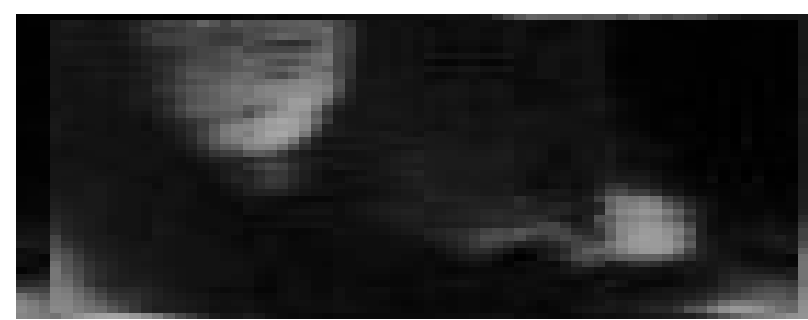

Fig. 5. (c) A normalized normal image of iris before enhancement. 


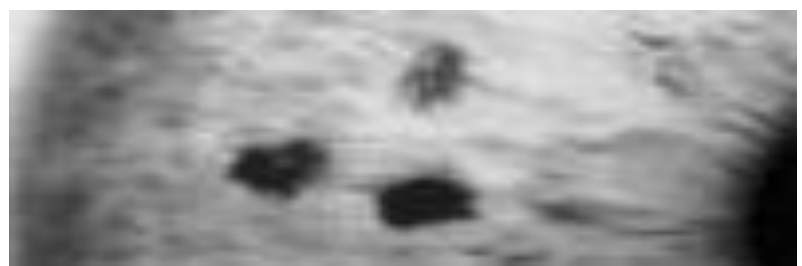

Fig. 5. (d) A normalized image of iris showing signs of broken tissues after enhancement.

\section{CONCLUSION}

For precise detection of diabetes the iris image showing the signs of presence of broken tissue was first analysed by the second parameter which is color change in the region of pancrease, and then sent to artificial neural network for classification and verification. Finally the result obtained from this system were compared with an insulin normality test for further verification. Amongst 10 patient tested from March to June 2014 in Tianjin Medical University, the system detected abnormality of pancreas to 8 patients and when subjected to insulin Normality test, all 8 patients were confirmed to have diabetes type II. Therefore it can be seen from the result that the system can detect abnormality of a pancreas organ with an accuracy of $100 \%$ by evaluating an image of the iris. Therefore iridology is an ideal prediagnostic tool.

In this research the detection of diabetes through the corresponding region of pancreas in the iris of the eye is based on color coding system and visual inspection. In future this detection method should be converted into some metric form. With metric form the diabetes status of the patient can be categorized as mild,moderate and severe.

\section{ACKNOWLEDGMENT}

Jamal Banzi thanks his father Mr. Firmat M Banzi for his full heart support and courage on all the matters concerning his studies and research. Special thanks also to his supervisor Professor Zhaojun Xue of Tianjin University of Technology and Education who gives direction and guidance in accomplishing this work. He also express his gratitude to Prof J. Anatory, Mr. H Mongi, Mr. J Woiso and Mr. Baraka Kondo all from University of Dodoma, Tanzania for their advice, courage, and editing and idea refinement of most of his research works.

\section{REFERENCES}

[1] F. Sharan, "Iridology A complete guide to diagnosing through the iris and to related forms of treatment," School of natural medicine Boulder CO 80306 USA, 1992.

[2] L. Robert, Opencv2computer Vision Application Programming Cookbook, Packt Publishing Ltd 32, Lincoln Road Olton Birmingham, B27 6PA, UK, 2011.

[3] L. Ma and N. Li, "Texture feature extraction and conference on medical biometrics," in Proc. ICMB, LNCS 4901, Hong Kong, 2008, pp. $168-175$.

[4] World Health Organization, "Use of glycated haemoglobin (HBA1C) in the diagnosis of diabetes mellitus," WHO publication 20 Avenue Appia, 1211 Geneva 27, Switzerland, 2011.

[5] A. D. Wibawa and M. H. Purnomo, "Early detection on the condition of pancreas organ as the cause of diabetes mellitus by real time iris image processing," in Proc. IEEE Asia Pacific Conference on Circuits and Systems, 2006, pp. 1008-1010

[6] D. Dunn, W. E. Higgins, and J. Wakeley, Texture Segmentation Using $2 D$ Gabor.

[7] J. Daugman, "New methods in iris recognition," IEEE Transactions on Systems, Man, and Cybernetics, Part B, vol. 37, pp. 1167-1175, 2007.

[8] U. M. Chaskar and M. S. Sutaone, "Learning to predict diabetes from iris image analysis," International Journal of Biomedical Engineering and Technology, vol. 9, no. 1, 2012.

[9] S. Haykin, Neural Networks and Learning Machines, Pearson Education Inc., 2009.

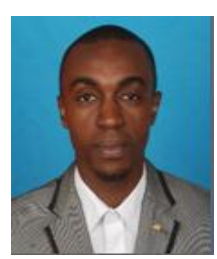

Jamal Firmat Banzi was born in Tanzania, East Africa. $\mathrm{He}$ received bachelor's degree of science in information systems from University of Dodoma, Tanzania. He then worked as a tutorial assistant/instructor for some months before he joined Tianjin University of Technology and Education in China for his further studies. He is currently pursuing his master's degree of science in signal and information processing engineering, Tianjin University of Technology and Education. His research interests include computer vision and pattern recognition, artificial neural networks and intelligent systems, computer assisted diagnosis of diseases based on image processing and Biomedical engineering.

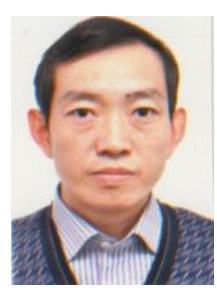

Zhaojun Xue received the bachelor's degree in mechanical design and automation at Shandong University, Jinan, China. He received his master's degree in biomedical engineering from Tongji University, Shanghai, China, and completed his doctor's degree in biomedical engineering from Tianjin University, Tianjin, China. $\mathrm{He}$ is presently a postdoctoral fellow in Tianjin University. His research interests include bioelectronics, biomedicine information detection, image processing, and pattern recognition. 